\title{
SHORT REPORT \\ Under-reporting giardiasis: time to consider the public health implications
}

\author{
S. L. CURRIE ${ }^{1}$, N. STEPHENSON ${ }^{2}$, A. S. PALMER ${ }^{3}$, B. L. JONES ${ }^{1}$ AND \\ C. L. ALEXANDER ${ }^{1 *}$ \\ ${ }^{1}$ Scottish Parasite Diagnostic and Reference Laboratory, Glasgow, Scotland, UK \\ ${ }^{2}$ Department of Microbiology, Aberdeen Royal Infirmary, NHS Grampian, Aberdeen, Scotland, UK \\ ${ }^{3}$ Gastrointestinal and Zoonoses Section, Health Protection Scotland, Glasgow, Scotland, UK
}

Received 11 May 2017; Final revision 7 July 2017; Accepted 10 August 2017;

first published online 7 September 2017

\section{SUMMARY}

Giardiasis is a treatable disease, caused by the flagellated protozoan parasite, Giardia duodenalis (G. duodenalis). It is one of the most common enteric parasites found globally to cause gastrointestinal disturbances, and infections may result in long-term irritable bowel syndrome-like symptoms. It is a common misconception that giardiasis is associated with foreign travel, which results in locally acquired cases in the UK being underdiagnosed. This report highlights the findings from one large Scottish Health Board, arising from a change in testing methodology, which resulted in the screening of all stools submitted for enteric investigations for $G$. duodenalis. Previous selection criteria were restricted to patients with a travel history to specific regions of the world, or on the basis of certain clinical details. In this report, clinical details were recorded from samples shown to be positive using two methods: an ELISA-based antigen detection assay and microscopy. Clinical details were assessed for a total of 28 laboratory-confirmed positive cases against the original selection criteria. Twenty-six cases (93\%) would have been excluded from Giardia testing if the previous selection criteria had been applied. Although nine cases stated foreign travel, only two had been to regions deemed to be 'high risk'. Therefore, those seven cases that travelled to perceived 'low-risk' regions would have been excluded from testing for this reason. This summary highlights the need for significant improvements to the selection criteria for Giardia testing. Laboratories should be encouraged towards the testing of all routinely submitted stools for this neglected pathogen to ensure cases that are acquired locally are properly identified and treated effectively.

Key words: Gastrointestinal infections, Giardia lamblia, giardiasis, parasites, public health microbiology.

\section{SHORT REPORT}

Giardia duodenalis (also referred to as Giardia lamblia or Giardia intestinalis) is a flagellated protozoan parasite, capable of causing giardiasis. After an incubation

\footnotetext{
* Author for correspondence: Dr C. L. Alexander, Scottish Parasite Diagnostic and Reference Laboratory, Glasgow G31 2ER, Scotland, UK.

(Email: Claire.Alexander@ggc.scot.nhs.uk)
}

period of between 5 and 25 days, patients present with gastrointestinal disturbances including diarrhoea, abdominal cramps, bloating, foul smelling belching and weight loss. In addition, the occurrence of longterm conditions including chronic fatigue and irritable bowel syndrome have been reported several years post-infection [1]. Although giardiasis may be selflimiting, it is a treatable disease and asymptomatic carriage is possible. Infection can occur in a number 
of hosts including livestock, wild and companion animals, as well as humans [2]. Different Giardia sub-types known as assemblages exist (A-H) of which assemblages $\mathrm{A}$ and $\mathrm{B}$ have the broadest host range and are the most commonly reported cause of giardiasis in humans [2]. Spread occurs via the faecal-oral route due to the consumption of food or water contaminated with infective cysts. Transmission by human or animal contact is possible with risk factors including visits to petting farms, owning dogs and contact with recreational or environmental water [3-5]. Good hygiene practice can limit spread of the disease through the washing of hands with soap and water after visiting the bathroom, changing nappies and before the preparation of food and drink.

In the UK, diagnostic testing methods are based on those stated in the UK Standards for Microbiological Investigation (SMI) B31 and S7 [6, 7]. As a consequence, only four out of 19 Scottish diagnostic laboratories currently test all stool specimens for Giardia. The majority of laboratories across Scotland select samples for Giardia examination based largely on travel history, due to the misconception that Giardia is acquired outwith the UK [8]. This results in only a small subset of samples being tested for this parasite. Therefore, the 10-year average of 199 laboratory reports per annum in Scotland is likely to be a significant under-reporting of the true number of cases [9]. This observation is also supported in other regions of the UK and in European countries, where it has previously been stated that disease burden is likely to be underestimated due to the lack of routine testing for Giardia [4, 10, 11]. A study examining Giardia reporting in Scotland in 2002 [12] found similar results and suggested that standardisation of Giardia testing across Scotland would enable comparative surveillance between Health Boards. Such a change would also highlight any differences in geographical dispersion of Giardia throughout Scotland.

A recent audit highlighted that the majority of laboratories in Scotland use microscopy to detect Giardia from stool samples [8]. However, in light of losing valuable microscopy expertise through an ageing workforce and a drive towards standardised automated methodologies, alternative detection methods are being considered. These include molecular panels and ELISA-based antigen assays (EIA) which detect multiple pathogens from a single stool [13]. A large NHS region, Grampian Health Board, was one of the first in Scotland to fully implement an EIA test to replace conventional microscopy detection for
Giardia. This enabled the rapid, automated testing of all stools, rather than a small subset. This report describes the impact of this change and highlights important findings, which demonstrate the need for major improvements to the selection criteria for Giardia testing.

During July 2015, a Giardia/Cryptosporidium Combo EIA kit (Launch Diagnostics IVD Research) was implemented for the routine testing of all stools submitted from human cases residing within NHS Grampian. Testing was performed on a total number of 5833 stools from 22 July to 29 December 2015 by the local NHS diagnostic microbiology laboratory, Aberdeen, UK. The EIA testing was performed according to the manufacturer's instructions. Briefly, $100 \mu \mathrm{l}$ of diluted stool sample $(0.1 \mathrm{~g}$ faecal matter in $0.7 \mathrm{ml}$ dilution buffer) was added to a sample well coated with anti-Giardia (and anti-Cryptosporidium) antibodies. After incubating and washing, anti-Giardia antibodies (and anti-Cryptosporidium) conjugated to horseradish peroxidase were added to each well. Following further incubation and washing, substrate (tetramethylbenzidine and peroxide) was added to each well prior to incubation for $10 \mathrm{~min}$ and the addition of stop solution. A colour change denoted a change in optical density (OD), which was read on an ELISA plate reader (filters $450 / 650 \mathrm{~nm}$ ) with positive ELISA values having an OD $>0 \cdot 08$. All EIA-positive samples were examined by bright-field microscopy for the presence of Giardia cysts. Confirmatory testing for both Giardia and Cryptosporidium was performed, when required, by the Scottish Parasite Diagnostic and Reference Laboratory (SPDRL).

For this report, we describe clinical data recorded on request forms, and laboratory data obtained from 28 samples shown to be positive by EIA testing and confirmed to be Giardia-positive by microscopy. Clinical data were retrieved from the laboratory requests forms submitted with each sample (Table 1). Only eight of the 28 EIA-positive samples had a history of recent travel outwith the UK noted in the clinical details section of the laboratory request form (Table 1). However, of those eight patients, five $(63 \%)$ would not have been considered for microscopy testing if based on the current SMIs as they had travelled to countries within the European Union, North America, Australia or New Zealand (Table 1). In addition to foreign travel to specific areas, NHS Grampian previously applied the following selection criteria when determining which samples to be tested for Giardia by microscopy: request forms specifically 
Table 1. Summary of the patient information provided on laboratory request forms for confirmed positive giardiasis cases

\begin{tabular}{ll}
\hline \hline Variable & Data \\
\hline Number of laboratory-confirmed cases & 28 \\
$\begin{array}{l}\text { Number of cases that would have been tested using the current } \\
\text { selection criteria based largely on recent travel to endemic regions }\end{array}$ & 3 \\
Male : female ratio & $17: 11$ \\
Age range & $3-77$ years \\
& $<17$ years $n=26$ cases \\
Symptoms & $>17$ years $n=2$ cases \\
& Loose stools $(n=6)$ \\
& Diarroea $(n=15)$ \\
& Loose stools and diarrhoea $(n=1)$ \\
& Diarroea, flatulence and vomiting $(n=5)$ \\
Countries visited (when stated) & No symptoms stated $(n=1)$ \\
& Azerbaijan $(n=1)$, Turkey $(n=2)$, Costa Rica $(n=1)$, \\
& Portugal $(n=2)$, Australia $(n=1)$, Thailand $(n=1)$ \\
\hline \hline
\end{tabular}

stating examination 'for ova, cysts and parasites'; those with previous giardiasis; immuno-compromised or immuno-suppressed patients; those with symptoms persisting for $>3$ months. This of course, relied on the information being provided on the request form by the sender clinician. If these specific criteria were adhered to, the majority of EIA-positive samples $(n=26 ; 93 \%)$ would not have been tested for Giardia. Of note was that not all those shown to be EIA-positive had diarrhoea recorded on the request form (Table 1). Other relevant clinical details included one case who had recently received treatment for giardiasis.

These results highlight several important findings: (a) the presence of Giardia in patients who did not have a recent history of foreign travel recorded on the laboratory request form, (b) the presence of Giardia in patients who had a recent travel history but to areas perceived to be 'low risk' for giardiasis. Therefore, these would be excluded from Giardia testing if using the most common selection criteria based largely on travel to specific 'high-risk' regions. However, the following details should be taken into account; with the lack of a national Giardia enhanced surveillance programme in the UK, valuable in-depth information on each case is lacking. In particular, travel details are provided on the request forms at the discretion of the requesting clinician and this information is often completely missing or incomplete. This can be misleading as some of the 28 cases may have travelled, but this information was not captured on the request form. In addition, geographical areas are stated to be low risk based on limited reporting of giardiasis. However, this may reflect the lack of countries having robust reporting/surveillance systems despite there being cases of giardiasis.

In the UK, giardiasis has been associated with risk factors other than travelling abroad, including children at nursery/playgroup and changing nappies [4]. Despite this link to children and childcare, 26 of the 28 positive stools were aged 17 years or above. A larger number of cases would have to be examined to determine if there is a greater likelihood of giardiasis in children. No details were available on any of the request forms regarding exposures. A number of risk factors have been identified including drinking tap water, swimming pools, eating lettuce and recreational fresh water contact $[4,14]$. In addition, there has been interest around companion animals as potential sources although transmission has been disputed [2]. A positive association between owning a dog and assemblage A infection has been described [4]. Further work is required to explore potential sources in animals, foods and environmental/recreational water within the UK.

The varying severity of giardiasis has been suggested to be linked to the assemblage type although the evidence is limited and conflicting. Minetti et al. found that assemblage B was more greatly associated with symptoms of vomiting, abdominal pain, swollen stomach and loss of appetite and a greater number of symptoms than assemblage A [4]. However, assemblage $\mathrm{A}$ has been associated with more severe diseases in another study [2]. A snapshot epidemiological study of Giardia assemblages in Scotland found that the majority of cases were infected with assemblage A but highlighted the importance of the host-parasite 
interaction in the clinical outcome [15]. Further, in-depth molecular studies are required to determine if the differences between symptoms reported in the 28 cases reviewed in this report are associated with differences in assemblage profiles.

Stool consistency can be used as a selection method for microbial pathogen testing. However, Lindo et al. found no association between faecal consistency and the rate of detection of Giardia cysts [16]. This is supportive of the findings within this report, where diarrhoea was not recorded in every positive case. However, this may reflect the lack of these data being included on each request form. It should also be noted that individuals can be asymptomatic or exhibit low-level symptoms despite harbouring infective cysts. Therefore, care should be taken when developing inclusion selection criteria, as diarrhoea may not always be an indicator of active infection and details of specific symptoms are often excluded from request forms.

Due to possible differences in the sensitivity and/or specificity of microscopy vs. antigen detection, 11 EIA-positive samples were found to be negative by microscopy. Those eleven samples were excluded as it could not be confirmed by the SPDRL if the EIA-positive findings were due to the presence of Cryptosporidium or Giardia since all confirmatory tests were negative. It cannot be ruled out that these are indeed true-positive findings as conventional microscopy has been reported to be less sensitive than EIA and molecular-based assays [12, 17]. There are situations where false negatives are reported using microscopy. These include cases where (a) parasitaemia is low, (b) the excretion of cysts is erratic or (c) microscopy expertise is lacking. An overview of sensitivity and specificity of diagnostic tests for giardiasis is reviewed by Minetti et al. [18].

Laboratory testing methods are decided at a local level, based on the SMIs. While it is suggested that Giardia should be examined in secondary testing, the SMI S7 recommends that Giardia should be added to the primary testing set where feasible. However, an audit of Scottish microbiology laboratories highlighted that only $2-18 \%$ of all stools received are examined for Giardia depending on the local health board resulting in the majority not being tested at all for this pathogen [8]. In light of health boards losing valuable microscopy expertise through a retiring population, considerations should be given to implementing rapid, cost-effective and simple alternatives to microscopy. These will permit a higher throughput to support the first line screening of all stools for Giardia, requiring less 'hands on' technical time and less expertise while improving detection sensitivity. Based on the data from this report, where approximately $60 \%$ of cases are not tested, and considering the total number of positive cases is approximately 200 per annum using the current 'recent travel' policy [9], one could speculate as many as 300 cases across Scotland are being missed.

In summary, this report highlights the need for significant improvements around Giardia selection criteria and testing algorithms at a national and international level. A Scottish Health Protection Network Giardia Short-Life Working Group has been established with expertise from Public Health, Health Protection Scotland, SPDRL and microbiologists to consider public health investigation and management of giardiasis cases on the assumption that laboratories will migrate towards the testing of all stools for Giardia in future. Although implementing more sensitive, automated testing procedures will depend on local resources including funding, staff provision and service demands, efforts should be made to encourage movement towards the testing of all stools. By ensuring both locally acquired cases and travel-related cases are accurately diagnosed, this will allow patients to promptly receive suitable treatment to relieve unpleasant symptoms and to minimise long-term sequelae. As more laboratories move towards the testing of all stools for Giardia, it is possible that outbreaks will become evident. Having an accurate diagnosis will therefore support further essential investigations to minimise spread of disease. In addition to encouraging the testing of all stools, further extensive molecular work is essential to explore possible sources, transmission routes, profiles and novel biomarkers to greatly improve our understanding of this neglected, yet significant pathogen.

\section{ACKNOWLEDGEMENTS}

The authors wish to express their thanks to the Healthcare Scientists in the Department of Microbiology, NHS Grampian for undertaking the practical aspects of new method validation.

\section{DECLARATION OF INTEREST}

None. 


\section{DISCLAIMERS}

None.

\section{REFERENCES}

1. Wensaas KA, et al. Irritable bowel syndrome and chronic fatigue 3 years after acute giardiasis: historic cohort study. Gut 2012; 61(2): 214-219. doi: 10.1136/ gutjnl-2011-300220. Epub 2011 Sep 12.

2. Feng Y, Xiao L. Zoonotic potential and molecular epidemiology of Giardia species and giardiasis. Clinical Microbiology Reviews 2011; 24: 110-140.

3. Adam EA, et al. Giardiasis outbreaks in the United States, 1971-2011. Epidemiology and Infection 2016; 144: 2790-2801.

4. Minetti C, et al. Case-control study of risk factors for sporadic giardiasis and parasite assemblages in Northwest England. Journal of Clinical Microbiology 2015; 53: 3133-3140.

5. Steinmuller N, et al. Outbreaks of enteric disease associated with animal contact: not just a foodborne problem anymore. Clinical Infectious Diseases 2006; 43: 1596-1602.

6. Public Health England. Investigation of specimens other than blood for parasites. UK Standards for Microbiology Investigations 2017; B 31: Issue 5 (https://www.gov.uk/ uk-standards-for-microbiology-investigations-smi-qualityand-consistency-in-clinical-laboratories). Accessed April 2017.

7. Public Health England. Gastroenteritis and Diarrhoea. UK Standards for Microbiology Investigations 2013; S 7: Issue 1 (https://www.gov.uk/government/collections/ standards-for-microbiology-investigations-smi). Accessed April 2017.

8. Alexander C, et al. An audit of Cryptosporidium and Giardia detection in Scottish National Health Service diagnostic microbiology laboratories. Epidemiology and Infection. 2017; 145: 1584-1590.

9. Health Protection Scotland GIZ Team. Protozoal infections: incidence of protozoal infection reported to HPS in 2015. HPS Weekly Report 2016; 37: 284-285.

10. Ellam H, et al. Surveillance of giardiasis in North-west England 1996-2006: impact of an enzyme immunoassay test. Eurosurveillance 2008; 37: 1-5.

11. European Centre for Disease Prevention and Control. Annual Epidemiological Report 2016 - Giardiasis. Stockholm: ECDC, 2016 (http://ecdc.europa.eu/en/ healthtopics/Giardiasis/Pages/Annual-epidemiologicalreport-2016.aspx). Accessed July 2017.

12. Pollock KGJ. A survey of reporting of Giardia spp. by Scottish microbiology laboratories. SCIEH Weekly Report 2002; 36: 211-212.

13. Soares R, Tasca T. Giardiasis: an update review on sensitivity and specificity of methods for laboratorial diagnosis. Journal of Microbiological Methods 2016; 129: 98-102.

14. Stuart JM, et al. Risk factors for sporadic giardiasis: a case-control study in South-western England. Emerging Infectious Diseases 2003; 9: 229-233.

15. Alexander C, et al. Genotyping of Giardia isolates in Scotland: a descriptive epidemiological study. Epidemiology and Infection 2014; 142: 1636-1639.

16. Lindo JF, et al. Epidemiology of giardiasis and cryptosporidiosis in Jamaica. American Journal of Tropical Medicine and Hygiene 1998; 59: 717-721.

17. Gotfred-Rasmussen $\mathbf{H}$, et al. Comparison of sensitivity and specificity of 4 methods for detection of Giardia duodenalis in feces: immunofluorescence and PCR are superior to microscopy of concentrated iodine-stained samples. Diagnostic Microbiology and Infectious Disease 2016; 84: 187-190.

18. Minetti C, et al. Clinical update on giardiasis. $B M J$ 2016; 355: 201-204. 